THE EFFECT OF REGOSOL SOIL MIXED MEDIA, CHARCOAL POWDER AND MANURE

ON THE SEEDLING GROWTH OF PINE (Pinus merkusii jungher de Vrieser)

Marningot Tua Natalis Situmorang

\title{
THE EFFECT OF REGOSOL SOIL MIXED MEDIA, CHARCOAL POWDER AND MANURE ON THE SEEDLING GROWTH OF PINE (Pinus merkusii Jungher de Vrieser)
}

\author{
(Pengaruh Media Campuran Tanah Regosol, Serbuk Arang dan Pupuk Kandang terhadap \\ Pertumbuhan Semai Pinus (Pinus merkusii Jungher De Vrieser) \\ Marningot Tua Natalis Situmorang \\ Department of Environmental Engineering, Faculty of Engineering, Universitas Sahid \\ Jl. Prof. Dr. Soepomo No.84, RT.7/RW.1, Menteng Dalam, Kota Jakarta Selatan; Jakarta, Indonesia \\ e-mail: marningotnatalis.usahid@gmail.com
}

Paper submitted: July $23^{\text {th }}, 2021$; Paper revised: October $15^{\text {th }}, 2021$; Paper accepted: December $18^{\text {th }}, 2021$

\begin{abstract}
ABSTRAK
This study aimed to determine whether mixed seedling media between regosol soil with manure and charcoal powder at various concentrations would affect seedlings growth of Pinus merkusii. This study used a completely randomized design (CRD) with six treatments and four replications: A: $100 \%$ regosol soil (control). B.: 50\% regosol soil $+50 \%$ manure, C: $50 \%$ regosol soil $+50 \%$ charcoal. D : $50 \%$ regosol soil + $25 \%$ manure $+25 \%$ charcoal, E: $25 \%$ regosol soil $+25 \%$ manure $+50 \%$ charcoal and F: $25 \%$ regosol soil $+50 \%$ manure $+25 \%$ charcoal. The parameters measured were seedling height and diameter that carried out once every 10 days until harvest. After harvesting (60 days old seedling), the fresh weight, the total dry weight and the ratio of shoots to roots was calculated. Statistical analysis were carried out with Analysis of Variance and continued with Duncan's test at the 5\% and 1\% confidence levels. The results showed the growth of seedlings of $P$. merkusii planted on a mixture of $50 \%$ regosol soil $+25 \%$ manure $+25 \%$ charcoal (media D) resulted in a highest growth of the seedlings that is $4.35 \mathrm{~cm}$, in average of height. The average diameter of the seedlings is $0.55 \mathrm{~mm}$, the fresh weight of the seedlings is $0.220 \mathrm{~g}$, the total dry weight of the seedlings is $0.035 \mathrm{~g}$, and the ratio of shoots to roots of seedlings is 1,26 .
\end{abstract}

Keywords: charcoal powder, media seedlings, manure, pine, regosol soil, seedlings

\begin{abstract}
Penelitian bertujuan untuk mengetahui apakah penggunaan media semai campuran antara tanah regosol dengan pupuk kandang dan serbuk arang pada berbagai konsentrasi akan berpengaruh terhadap pertumbuhan semai $\underline{\mathrm{P}}$. merkusii. Penelitian ini menggunakan Rancangan Acak Lengkap (RAL) dengan enam perlakuan dan empat kali ulangan, yaitu : A : Tanah regosol $100 \%$ (Kontrol). B. : Tanah regosol $50 \%+$ Pupuk kandang $50 \%$, C : Tanah regosol $50 \%+$ arang $50 \%$. D : Tanah regosol $50 \%+$ pupuk kandang 25 $\%+\operatorname{arang} 25 \%$, E : Tanah regosol $25 \%+$ pupuk kandang $25 \%+$ arang $50 \%$ dan F : Tanah regosol 25 $\%+$ pupuk kandang $50 \%+$ arang $25 \%$. Parameter yang diukur adalah tinggi dan diameter dilakukan setiap 10 hari sekali hingga panenan, setelah panenan (umur bibit 60 hari) ditimbang berat basah semai, berat kering total semai dan dihitung rasio pucuk akar semai. Analisis data menggunakan Analisa Ragam dan dilanjutkan dengan uji Duncan pada tingkat kepercayaan $5 \%$ dan $1 \%$. Hasil penelitian menunjukkan pertumbuhan semai $P$. merkusii yang ditanam pada media campuran tanah regosol $50 \%+$ pupuk kandang $25 \%+$ arang $25 \%$ (media D) menghasilkan pertumbuhan semai tertinggi dengan tinggi rata-rata semai sebesar 4,35 cm, diameter rata-rata semai sebesar 0,55 mm, berat basah semai 0,220 gr, berat kering total semai 0,035 gr dan rasio pucuk akar semai sebesar 1,26.
\end{abstract}

Kata kunci: serbuk arang, media semai, pupuk kandang, pinus, tanah regosol, semai

\section{INTRODUCTION}

The government's policy to develop timber estate (HTI) on a large scale is one of the important programs which is priority in forestry development. This program is intended to increase the potenty of production forests as a source of supply of raw materials for the timber industry and to expand employment. Today, the domestic demand for wood carpentry, pulp, and energy is increasing in line with the development of the population and progress.

Author contributions: Marningot Tua Natalis Situmorang is the main contributor of the work and this paper 
Until 2020 the demand for wood for domestic consumption is estimated at 70 million $\mathrm{m}^{3} /$ year (Hayati, M., \& Hayati, 2015).

The need for such wood is impossible to fulfill if we rely solely on natural production forest covering an area of 64 million hectar, which until now has a low potential. In addition to the fact that there is around 15 million hectar of unproductive forest in permanent production forest areas in the form of open forest, shrubs, Imperata, and bare land with a productivity of about 0.5 $\mathrm{m}^{3} /$ year -1 $\mathrm{m}^{3} /$ year (Kuswiyono \& Qomar, 2020).

To meet future timber needs, HTI development is a priority that needs to be supported. One of the important aspects that must be considered in the development of timber estate is selecting the suitable types of plants and their fast growth, known characteristics, and mastery of cultural technology. One of the species chosen is Pinus merkusii Jungher de Vriese. It does not require high life requirements, grows fast, and has versatile benefits (Fachreza, 2021). And also it can be used to building wood, paper industry, and gondorukem (Sallata, 2013).

In general, the procurement of forest tree seeds through nurseries uses topsoil media is necesssary. However, this causes several problems, including the rich, not cohesive media so that it breaks easily, the root system is not compact, aeration and water percolation are not good, and it causes erosion. For this reason, it is necessary to find alternative media that can overcome this problem.

This study attempted to mix regosol soil with charcoal powder and manure at several composition as seedling material based on the problems above. This study aimed to determine whether mixed seedling media between regosol soil with manure and charcoal powder at various concentrations would affect seedlings growth of Pinus merkusii.

\section{MATERIALS AND METHODS}

\section{A. Materials and Equipment}

The study was conducted in a greenhouse and silviculture laboratory, Faculty of Biology, Pakuan University Bogor. The time required for the research was approximately three months, starting from September $13^{\text {th }}$ to December $12^{\text {th }}$ of 2020 , covering half a month of preparatory activities and two-and-a-half months of observation. The materials used in this study were: seeds of $P$. merkusii taken from Pine seed sources of RPH Cijambu BKPH East Manglayang KPH Sumedang Perum Perhutani Unit III West Java, seedling media (including regosol soil, charcoal 
powder and manure, sand, water). The tools used are: germination tub, tub mixing, media mix, polybag, sprayer, tweezers, sand and soil filter, bucket, thermometer, label, height gauge, diameter gauge (caliper), measuring cup, stationery, calculator, oven, and scales.

\section{B. Research Procedure}

\section{Germination}

P. merkusii seeds to be germinated were healthy and intact. The seeds were soaked in water for 24 hours; after that, the seeds were sown in a seedbed and maintained until they were 20 days old to be transferred to polybags. The polybag size used has a length of $15 \mathrm{~cm}$, a diameter of $10 \mathrm{~cm}$. The medium used for seed germination P. merkusii was a mixture of soil and sand $(1: 1, \mathrm{v} / \mathrm{v})$, roasted in advance with a heater for approximately 1 hour. Then the media is cooled and then put into seedbed in the greenhouse.

\section{Preparation of Seedling Media}

The regosol soil used is first sieved then roasted. The regosol soil was taken from hilly soil in the Cimalaka Sumedang area. Cow dung to be used was brown decomposed material air-dried under the sun and sieved using a filter. Cow dung was collected from a cow farm of Padjadjaran University, Jatinangor. Powder direction used is obtained by pounding until soft charcoal, and sifted using a filter. After all the media was ready, mixing is done between regosol soil, manure, and charcoal with different compositions. The media was then put into a polybag and labeled them according to the given treatments.

\section{Research Implementation}

Implementation of the research consisted of:

a. Weaning

P.merkusii seedlings of 20 days old weaned into a black plastic bag by way of the media sand seedling poke which had previously been moistened with water, then the seedlings were removed so that the roots of the seedlings were not damaged. The selected seedlings must be healthy and of uniform size, then planted in polybags containing media and placed in a greenhouse during the study.

\section{b. Maintenance}

During this study, the maintenance carried out was watering every day in the morning and evening, controlling weeds, pests, and diseases.

c. Observations

Observations made during the study included the measurement of seedling height, seedling diameter, fresh seedling weight, dry seedling weight, root shoot ratio.

1) Seedling Height

Measurement of seedling height was carried out after the seedlings were 20 days old in polybags. Subsequent measurements were made every 10 days until the end of the study. Height measurement was carried out using a ruler, starting from the base of the stem to the highest growing point on the stem. The increase in height is the difference in height at 
Jurnal Perbenihan Tanaman Hutan

Vol.9 No2 Desember 2021: 143-156

p-ISSN : 2354-8568

e-ISSN : 2527-6565

the final measurement minus the initial measurement.

2) Seedling Diameter.

The first measurement of seedling diameter was carried out simultaneously with the measurement of seedling height and repeated every 10 days until the end of the study; diameter measurements were carried out using calipers at the stem base. The increase in seedling diameter is the difference in diameter at the final measurement minus the initial measurement.

3) Fresh Weight Seedling

fresh weight was obtained by weighing all parts of the seedling in a fresh state, carried out at the end of the study.

\section{4) Dry Weight Seedling}

First the roots and shoots of seedlings were separated then heated in the oven for 48 hours at $80^{\circ} \mathrm{C}$ until reaching a constant weight, and then weighed.

\section{5) Shoot-Root Ratio}

Shoot-ratio ratio is the ratio of shoot dry weight and seedling root dry weight.

\section{Data Analysis}

This study used a completely randomized design with one control and five treatments. Each treatment was repeated four times. The treatment composition was as follows:
$\mathrm{A}=100 \%$ regosol soil (control)
$\mathrm{B}=50 \%$ regosol soil $+50 \%$ manure
$\mathrm{C}=50 \%$ regosol soil $+50 \%$ charcoal powder
$\mathrm{D}=50 \%$ regosol soil $+25 \%$ manure + charcoal powder $25 \%$
$\mathrm{E}=$ regosol soil $25 \%+$ manure $25 \%+$ charcoal powder $50 \%$
$\mathrm{F}=$ regosol soil $25 \%+$ manure $50 \%+$ charcoal powder $25 \%$

\section{RESULTS AND DISCUSSION}

\section{A. Results}

\section{Seedling Height}

An increase is a difference in height at the last measurement minus the initial measurement. The calculation of the analysis of variance followed by Duncan's test in height increment are presented in Table 1 and Table 2. During 60 days of observation (2 months old seedlings), the value of height increment is also presented in Table 2.

Table 1 shows that various media gave a significant effect on the height increament of $P$ merkusii seedlings. Based on the results of the above analysis, Duncan's distance test and percentage height increment toward control for the average growth of seedling height is presented in Table 2. 
Table (Tabel) 1. Analisys of variance (ANOVA) of the average height increment (cm) of P.merkusii seedling at the age of 60 days. (Analisis ragam rata-rata pertambahan tinggi semai $\underline{\mathrm{P}}$ merkusii pada umur 60 hari).

\begin{tabular}{|c|c|c|c|c|c|c|}
\hline \multirow[t]{2}{*}{$\begin{array}{l}\text { Source of variances } \\
\text { (Sumber keragaman) }\end{array}$} & \multirow{2}{*}{$\begin{array}{c}\text { Degree of } \\
\text { freedom/DF } \\
\text { (Derajat } \\
\text { bebas/(DB) }\end{array}$} & \multirow{2}{*}{$\begin{array}{c}\text { Sum } \\
\text { square/SS } \\
\text { (Jumlah } \\
\text { kuadrat/JK) }\end{array}$} & \multirow{2}{*}{$\begin{array}{c}\text { Mean sum } \\
\text { square/MSS } \\
\text { (Jumlah } \\
\text { kuadrat } \\
\text { tengah } / \mathrm{KT})\end{array}$} & \multirow[t]{2}{*}{$\begin{array}{c}\text { F count } \\
(F \text { Hitung })\end{array}$} & \multicolumn{2}{|c|}{$\begin{array}{c}\text { F table } \\
(F \text { Tabel })\end{array}$} \\
\hline & & & & & $5 \%$ & $1 \%$ \\
\hline Treatment (perlakuan) & 5 & 17.23 & 3.45 & $8.2 * *$ & 2.77 & 4.25 \\
\hline Error (galat) & 18 & 7.56 & 0,42 & & & \\
\hline Sum (jumlah) & 23 & 24,79 & & & & \\
\hline
\end{tabular}

Table (Tabel) 2. Duncan test of average height increment $(\mathrm{cm})$ and percentage toward control from each treatments of P.Merkusii seedling at 60 days old (Uji Duncan rata-rata pertambahan tinggi $(\mathrm{cm})$ dan persen peningkatan terhadap kontrol pada semai $\underline{\mathrm{P}}$. Merkusii pada umur 60 hari).

\begin{tabular}{|c|c|c|}
\hline $\begin{array}{l}\text { Media/Treatments } \\
\text { (Media/perlakuan) }\end{array}$ & $\begin{array}{c}\text { Average height increment } \\
\text { (Rata-rata Pertambahan } \\
\text { Tinggi })(\mathrm{cm})\end{array}$ & $\begin{array}{c}\% \text { of height increment toward control } \\
(\% \text { Peningkatan tinggi terhadap } \\
\text { Kontrol })\end{array}$ \\
\hline $\begin{array}{l}\mathrm{D}(50 \% \text { regosol soil }+25 \% \text { manure }+ \text { charcoal } \\
\text { powder } 25 \% / 50 \% \text { tanah regosol }+25 \% \\
\text { pupuk kandang }+25 \% \text { arang })\end{array}$ & $4.35 \mathrm{a}$ & 148.57 \\
\hline $\begin{array}{l}\mathrm{F}(25 \% \text { regosol soil }+50 \% \text { manure }+25 \% \\
\text { charcoal powder } 125 \% \text { tanah regosol }+50 \% \\
\text { pupuk kandang }+25 \% \text { arang })\end{array}$ & $3.85 \mathrm{ab}$ & 120 \\
\hline $\begin{array}{l}\mathrm{B}(50 \% \text { regosol soil }+50 \% \text { manure/ } 50 \% \text { tanah } \\
\text { regosol }+50 \% \text { pupuk kandang })\end{array}$ & $3.1 \mathrm{c}$ & 77.14 \\
\hline $\begin{array}{l}\text { E }(25 \% \text { regosol soil }+25 \% \text { manure }+50 \% \\
\text { charcoal powder } 25 \% \text { tanah regosol }+25 \% \\
\text { pupuk kandang }+50 \% \text { arang })\end{array}$ & $2.73 \mathrm{~d}$ & 56 \\
\hline $\begin{array}{c}\mathrm{C}(50 \% \text { regosol soil }+50 \% \text { charcoal powder } / \\
50 \% \text { tanah regosol }+50 \% \text { arang })\end{array}$ & $2.63 \mathrm{de}$ & 50.29 \\
\hline $\begin{array}{l}\text { A ( } 100 \% \text { regosol soil or control } / 100 \% \text { tanah } \\
\text { regosol atau kontrol) }\end{array}$ & $1.75 \mathrm{f}$ & \\
\hline
\end{tabular}

Media D (50\% Regosol soil + 25\% manure $+25 \%$ charcoal powder) shows a very significant effect on media B, E, C and A, and significantly different from media F. This indicated that the improvement in the composition of the growing media gave an increase in the growth of seedling height when compared to soil media (control), where the average height growth in D media was about $4.35 \mathrm{~cm}$, while control or in A media (soil) only $1.75 \mathrm{~cm}$. Thus media D can increase the growth of seedling height by $148.57 \%$ from control.

\section{Seedling Diameter}

An increase in seedling diameter is the difference between the last diameter measurement minus the initial measurement. Calculation of the analysis of variance in diameter increment is presented in Table 3. 
Table (Tabel) 3. Analysis of Variance of average diameter increment ( $\mathrm{mm}$ ) of P.merkusii seedlings at 60 days of age (Analisis keragaman pertambahan diameter rata-rata (mm) semai P.merkusii)

\begin{tabular}{lcccccc}
\hline $\begin{array}{c}\text { Source of variances } \\
\text { (Sumber keragaman) }\end{array}$ & $\begin{array}{c}\text { Degree of } \\
\text { freedom/D } \\
\text { F (Derajat } \\
\text { bebas /DB) }\end{array}$ & $\begin{array}{c}\text { Sum } \\
\text { square/SS } \\
\text { (Jumlah } \\
\text { kuadrat/ JK) }\end{array}$ & $\begin{array}{c}\text { Mean sum } \\
\text { square/MSS } \\
\text { (Jumlah kuadrat } \\
\text { tengah } / \mathrm{KT})\end{array}$ & $\begin{array}{c}\text { F count } \\
(F\end{array}$ & $\begin{array}{c}\text { F table } \\
(\text { F tabel })\end{array}$ \\
5 Treatment (perlakuan) & 5 & 0.18 & 0.036 & $4.348^{* *}$ & 2.75 & 4.25 \\
\hline Error (galat) & 18 & 0.15 & 0.0083 & & & \\
\hline Sum (jumlah) & 23 & 0.33 & & & & \\
\hline
\end{tabular}

Remark (Keterangan): ** High significant effect at level 1\% (Berpengaruh sangat nyata pada taraf $1 \%$ )

Analysis of variance (Table 3), shows that different growing media have a very significant effect on the increase in seedling diameter.
Duncan's test for the average diameter increase of seedlings is presented in Table 4, while the Duncan test calculation of the average diameter increase is in Table 4.

Table (Table)4. Duncan's test of the average diameter increment (mm) of P.merkusii seedlings at the age of 60 days (Uji Duncan rata-rata pertambahan diameter $(\mathrm{mm})$ semai $\underline{\mathrm{P}}$. merkusii pada umur 60 hari).

\begin{tabular}{|c|c|c|}
\hline $\begin{array}{l}\text { Media/Treatments } \\
\text { (Media/perlakuan) }\end{array}$ & $\begin{array}{c}\text { Average diameter } \\
\text { increment } \\
\text { (Rata-rata pertambahan } \\
\text { diameter })(\mathrm{mm})\end{array}$ & $\begin{array}{c}\% \text { of diameter increment toward } \\
\text { control } \\
(\% \text { Peningkatan diameter } \\
\text { terhadap kontrol })\end{array}$ \\
\hline $\begin{array}{l}\mathrm{D}(50 \% \text { regosol soil }+25 \% \text { manure }+ \text { charcoal } \\
\text { powder } 25 \% / 50 \% \text { tanah regosol }+25 \% \text { pupuk } \\
\text { kandang }+25 \% \text { arang })\end{array}$ & $0.55 \mathrm{a}$ & 83.3 \\
\hline $\begin{array}{l}\mathrm{F}(25 \% \text { regosol soil }+50 \% \text { manure }+25 \% \text { charcoal } \\
\text { powder } / 25 \% \text { tanah regosol }+50 \% \text { pupuk } \\
\text { kandang }+25 \% \text { arang })\end{array}$ & $0.55 \mathrm{a}$ & 83.3 \\
\hline $\begin{array}{l}\mathrm{B}(50 \% \text { regosol soil }+50 \% \text { manure/ } 50 \% \text { tanah } \\
\text { regosol }+50 \% \text { pupuk kandang })\end{array}$ & $0.49 \mathrm{~b}$ & 63.3 \\
\hline $\begin{array}{l}\mathrm{E}(25 \% \text { regosol soil }+25 \% \text { manure }+50 \% \\
\text { charcoal powder } 25 \% \text { tanah regosol }+25 \% \\
\text { pupuk kandang }+50 \% \text { arang })\end{array}$ & $0.49 \mathrm{~b}$ & 63.3 \\
\hline $\begin{array}{l}\mathrm{C}(50 \% \text { regosol soil }+50 \% \text { charcoal powder/ } 50 \% \\
\text { tanah regosol }+50 \% \text { arang })\end{array}$ & $0.43 \mathrm{c}$ & 43.3 \\
\hline $\begin{array}{l}\text { A ( } 100 \% \text { regosol soil or control/100\% tanah } \\
\text { regosol atau kontrol })\end{array}$ & $0.30 \mathrm{~d}$ & \\
\hline
\end{tabular}

Remark (Ketrangan): The numbers followed by the same letter and column are not significantly different (Angka yang diikuti oleh huruf yang sama menyatakan tidak berbeda nyata)

Duncan test results (Table 4) show that media D $(50 \%$ regosol soil $+25 \%$ manure + charcoal powder 25\%) and media F $(25 \%$ regosol soil $+50 \%$ manure + charcoal powder
$25 \%$ ) were significantly different from A, B, C and $\mathrm{E}$ media. Furthermore, it shows that improving the composition of the media increased the growth of seedling diameter. The 
average seedling diameter on media $\mathrm{D}$ and $\mathrm{F}$ can reach $0.55 \mathrm{~mm}$, while on media A (soil), $0.3 \mathrm{~mm}$ or an increase of $83.3 \%$. from control (A)

\section{Fresh weight of seedlings}

The analysis of variance for fresh weight of $P$. merkusii at 60 days old seedling is presented in Table 5.

Table (Tabel) 5. Analysis of variance (ANOVA) of average fresh weight P. merkusii seedlings at the age of 60 days (Analisis ragam rata-rata berat basah-semai $\underline{\mathrm{P} .}$ merkusii pada umur 60 hari).

\begin{tabular}{|c|c|c|c|c|c|c|}
\hline \multirow[t]{2}{*}{$\begin{array}{l}\text { Source of variance } \\
\text { (Sumber keragaman) }\end{array}$} & \multirow{2}{*}{$\begin{array}{c}\text { Degree of } \\
\text { Freedom/DF } \\
(\text { Derajat } \\
\text { bebas })(\mathrm{DB})\end{array}$} & \multirow{2}{*}{$\begin{array}{c}\text { Sum } \\
\text { Square/SS } \\
\text { (Jumlah } \\
\text { kuadrat/JK } \\
\text { ) }\end{array}$} & \multirow{2}{*}{$\begin{array}{c}\text { Mean Sum } \\
\text { Square/MS } \\
\mathrm{S} \text { (Jumlah } \\
\text { kuadrat } \\
\text { tengah } / \mathrm{KT})\end{array}$} & \multirow[t]{2}{*}{$\begin{array}{c}\text { F count } \\
(F \text { hitung })\end{array}$} & \multicolumn{2}{|c|}{$\begin{array}{c}\text { F Table } \\
(\text { F Tabel })\end{array}$} \\
\hline & & & & & $5 \%$ & $1 \%$ \\
\hline Treatmen (Perlakuan) & 5 & 0.05 & 0.01 & $1.59^{\mathrm{tn}}$ & 2.77 & 4.25 \\
\hline Error (Galat) & 18 & 0.113 & 0.0063 & & & \\
\hline Sum (Jumlah) & 23 & 0.163 & & & & \\
\hline
\end{tabular}

Based on analysis of variance (Table 5), give a significant effect on the fresh weight of indicating that different growth media did not seedlings of P. merkusii at 60 days old.

Table (Tabel) 6. Average fresh weight (gr) of P.merkusii seedlings at the age of 60 days (Rata rata berat basah (gr) semai P.merkusii pada umur 60 hari)

\begin{tabular}{|c|c|c|}
\hline $\begin{array}{l}\text { Media/treatments } \\
\text { (Media/perlakuan) }\end{array}$ & $\begin{array}{c}\text { Average of seedling fresh } \\
\text { weight (gr) (Rata-rata berat } \\
\text { basah semai }(\text { gr)) }\end{array}$ & $\begin{array}{l}\% \text { fresh weight increment } \\
\text { toward control (\% peningkatan } \\
\text { berat basah terhadap kontrol) }\end{array}$ \\
\hline $\begin{array}{l}\mathrm{D}(50 \% \text { regosol soil }+25 \% \text { manure }+ \text { charcoal } \\
\text { powder } 25 \% / 50 \% \text { tanah regosol }+25 \% \\
\text { pupuk kandang }+25 \% \text { arang })\end{array}$ & 0.220 & 185.71 \\
\hline $\begin{array}{l}\mathrm{F}(25 \% \text { regosol soil }+50 \% \text { manure }+25 \% \\
\text { charcoal powder } / 25 \% \text { tanah regosol }+50 \% \\
\text { pupuk kandang }+25 \% \text { arang })\end{array}$ & 0.170 & 120.78 \\
\hline $\begin{array}{l}\text { B }(50 \% \text { regosol soil }+50 \% \text { manure/ } 50 \% \text { tanah } \\
\quad \text { regosol }+50 \% \text { pupuk kandang })\end{array}$ & 0.159 & 106.49 \\
\hline $\begin{array}{l}\text { E }(25 \% \text { regosol soil }+25 \% \text { manure }+50 \% \\
\text { charcoal powder } 25 \% \text { tanah regosol }+25 \% \\
\text { pupuk kandang }+50 \% \text { arang })\end{array}$ & 0.153 & 98.70 \\
\hline $\begin{array}{c}\mathrm{C}(50 \% \text { regosol soil }+50 \% \text { charcoal powder/ } \\
50 \% \text { tanah regosol }+50 \% \text { arang })\end{array}$ & 0.125 & 62.34 \\
\hline $\begin{array}{l}\text { A (100\% regosol soil or control } / 100 \% \text { tanah } \\
\text { regosol atau kontrol) }\end{array}$ & 0.077 & \\
\hline
\end{tabular}

Table 6 shows that media D $(50 \%$ regosol soil $+25 \%$ manure $+25 \%$ charcoal powder) was the highest value of fresh weight of seedling but not significantly different from other media. This shows (Table 6) that the improvement in the composition of the media increased the fresh weight of the seedlings, where the average fresh weight of medium $\mathrm{D}$, seedlings could reach $0.220 \mathrm{~g}$, and media A or control (soil) is $0.077 \mathrm{~g}$ or an increase of $185.71 \%$ from control.

\section{Total dry weight of seedlings.}


Analysis of variance of the average of total dry weight of $P$. merkusii seedling is presented in Table 7, while the average value and percentage increment can be seen in Table 8.

Table (Tabel) 7. Analysis of variety (ANOVA) average total dry weight (gr) of p.merkusii seedlings at 60 days of age (Analisa ragam (ANOVA) rata-rata berat kering total (gr) semai P.merkusii pada umur 60 hari.)

\begin{tabular}{|c|c|c|c|c|c|c|}
\hline \multirow[t]{2}{*}{$\begin{array}{c}\text { Source of variance } \\
\text { (Sumber keragaman) }\end{array}$} & \multirow{2}{*}{$\begin{array}{c}\text { Degree of } \\
\text { Freedom/DF } \\
\text { (Derajat } \\
\text { Bebas) (DB) }\end{array}$} & \multirow{2}{*}{$\begin{array}{c}\text { Sum } \\
\text { Square/SS } \\
\text { (Jumlah } \\
\text { Kuadrat/JK) }\end{array}$} & \multirow{2}{*}{$\begin{array}{c}\text { Mean Sum } \\
\text { Square/MSS } \\
\text { (Jumlah Kuadrat } \\
\text { Tengah /KT) }\end{array}$} & \multirow[t]{2}{*}{$\begin{array}{c}\text { F count } \\
(F \text { hitung })\end{array}$} & \multicolumn{2}{|c|}{$\begin{array}{c}\text { F Table } \\
(F \text { tabel })\end{array}$} \\
\hline & & & & & $5 \%$ & $1 \%$ \\
\hline Treatment (perlakuan) & 5 & 0.001 & 0.0002 & $1.82 *$ & 2.77 & 4.25 \\
\hline Error (galat) & 18 & 0.002 & 0.00011 & & & \\
\hline Sum (jumlah) & 23 & 0.003 & & & & \\
\hline
\end{tabular}

Analysis of variance (Table 8), showed that different media have not a significant effect on the average total dry weight of $P$. Merkusii at 60 days old seedling The average total dry weight of seedlings on various media treatments compared to controls can be seen in
Table 9. The highest total dry weight occurred in media $\mathrm{D}$ which was 0.035 grams (increase of $180 \%$ compared to control media), followed by media F, B, E, C, and A, which were 0.028 grams, 0.026 grams, 0,024 grams, 0.020 grams, and 0.017 grams respectively.

Table (Tabel) 8. Average total dry weight (gr) of seedlings. P. merkusii at the age of 60 days (Rata - rata berat kering total (gr) Semai. P.merkusii pada umur 60 hari.)

\begin{tabular}{|c|c|c|}
\hline $\begin{array}{l}\text { Media/ Treatments } \\
\text { (Media/perlakuan) }\end{array}$ & $\begin{array}{l}\text { Average of Total Dry Weight } \\
\text { (Rata - rata berat } \\
\text { kering Total })(\mathrm{gr})\end{array}$ & $\begin{array}{l}\% \text { Increment toward } \\
\text { Control }(\% \\
\text { Peningkatant terhadap } \\
\text { kontrol })\end{array}$ \\
\hline $\begin{array}{l}\mathrm{D}(50 \% \text { regosol soil }+25 \% \text { manure }+ \text { charcoal } \\
\text { powder } 25 \% / 50 \% \text { tanah regosol }+25 \% \\
\text { pupuk kandang }+25 \% \text { arang })\end{array}$ & 0.035 & 180 \\
\hline $\begin{array}{l}\mathrm{F}(25 \% \text { regosol soil }+50 \% \text { manure }+25 \% \\
\text { charcoal powder } / 25 \% \text { tanah regosol }+50 \% \\
\text { pupuk kandang }+25 \% \text { arang })\end{array}$ & 0.028 & 64.71 \\
\hline $\begin{array}{l}\mathrm{B}(50 \% \text { regosol soil }+50 \% \text { manure/ } 50 \% \text { tanah } \\
\text { regosol }+50 \% \text { pupuk kandang })\end{array}$ & 0.026 & 52.94 \\
\hline $\begin{array}{l}\text { E }(25 \% \text { regosol soil }+25 \% \text { manure }+50 \% \\
\text { charcoal powder } 25 \% \text { tanah regosol }+25 \% \\
\text { pupuk kandang }+50 \% \text { arang })\end{array}$ & 0.024 & 41.18 \\
\hline $\begin{array}{l}\mathrm{C}(50 \% \text { regosol soil }+50 \% \text { charcoal powder/ } \\
50 \% \text { tanah regosol }+50 \% \text { arang }\end{array}$ & 0.020 & 17.65 \\
\hline $\begin{array}{l}\text { A }(100 \% \text { regosol soil or control/100\% tanah } \\
\text { regosol atau kontrol) }\end{array}$ & 0.017 & \\
\hline
\end{tabular}




\section{Shoot-root ratio}

The shoot ratio was obtained by comparing the dry weight of the shoots of the seedlings with the dry weight of the roots of the seedlings. Shoot-root ratio data for P.merkusii seedlings for each treatment. Analysis of shoot-root ratio variance is presented in table 9 .

Table (Tabel) 9. Analysis of variance (ANOVA) average shoot-root ratio of $P$.merkusi at the age of 60 days (Analisa ragam (ANOVA) rata-rata rasio pucuk-akar semai P.merkusi pada umur 60 hari).

\begin{tabular}{|c|c|c|c|c|c|c|}
\hline \multirow{2}{*}{$\begin{array}{c}\text { Source of variance } \\
\text { (Sumber keragaman) }\end{array}$} & \multirow{2}{*}{$\begin{array}{c}\text { Degree of } \\
\text { freedom/DF } \\
(\text { Derajat } \\
\text { bebas) }(\mathrm{DB})\end{array}$} & \multirow{2}{*}{$\begin{array}{c}\text { Sum } \\
\text { square/SS } \\
\text { (Jumlah } \\
\text { kuadrat/JK) }\end{array}$} & \multirow{2}{*}{$\begin{array}{c}\text { Mean sum } \\
\text { square/MSS } \\
\text { (Jumlah kuadrat } \\
\text { tengah } / \mathrm{KT})\end{array}$} & \multirow{2}{*}{$\begin{array}{l}\text { F count } \\
(F \text { hitung })\end{array}$} & \multicolumn{2}{|c|}{$\begin{array}{l}\text { F Table } \\
(F \text { tabel })\end{array}$} \\
\hline & & & & & $5 \%$ & $1 \%$ \\
\hline Treatment (Perlakuan) & 5 & 15.17 & 3.23 & $1.13 *$ & 2.77 & 4.25 \\
\hline Error (Galat) & 18 & 51.22 & 2,85 & & & \\
\hline Total (Total) & 23 & 67.39 & & & & \\
\hline
\end{tabular}

Analisys of variance (Table 9), indicating that the different types of media did not give a significant response to the average shoot-root ratio of seedlings.
The average value of shoot-root ratio and percentage increment toward control of seedlings is presented in table 10 .

Table (Tabel) 10. Average shoot-root ratio of $P$. merkusii seedlings at the age of 60 days (Rata- rata Rasio pucuk-akar semai P. merkusii pada umur 60 hari.

\begin{tabular}{|c|c|}
\hline $\operatorname{Media}($ Media $)$ & $\begin{array}{l}\text { Average shoot root ratio (Rata } \\
\text { - rata nilai rasio pucuk akar) }\end{array}$ \\
\hline A ((100\% regosol soil or control $/ 100 \%$ tanah regosol atau kontrol) & 2.94 \\
\hline B (50\% regosol soil $+50 \%$ manure/ $50 \%$ tanah regosol $+50 \%$ pupuk kandang) & 2.76 \\
\hline $\mathrm{C}(50 \%$ regosol soil $+50 \%$ charcoal powder/ $50 \%$ tanah regosol $+50 \%$ arang) & 1.80 \\
\hline $\begin{array}{l}\mathrm{D}(50 \% \text { regosol soil }+25 \% \text { manure }+ \text { charcoal powder } 25 \% / 50 \% \text { tanah } \\
\text { regosol }+25 \% \text { pupuk kandang }+25 \% \text { arang })\end{array}$ & 1.26 \\
\hline $\begin{array}{l}\mathrm{E}(25 \% \text { regosol soil }+25 \% \text { manure }+50 \% \text { charcoal powder } / 25 \% \text { tanah } \\
\text { regosol }+25 \% \text { pupuk kandang }+50 \% \text { arang })\end{array}$ & 0.97 \\
\hline $\begin{array}{l}\mathrm{F}(25 \% \text { regosol soil }+50 \% \text { manure }+25 \% \text { charcoal powder } / 25 \% \text { tanah regosol } \\
\quad+50 \% \text { pupuk kandang }+25 \% \text { arang })\end{array}$ & 0.88 \\
\hline
\end{tabular}

Table 10, shows that media A was very significantly different from media D, F, C, and $\mathrm{E}$ and significantly different from media B. However, the growth of seedlings on media A showed abnormal growth, meaning that the dry weight of shoots was much greater than the dry weight of the roots, even though the total dry weight of seedlings was very small when compared to the total dry weight of other media.

\section{B. Discussion}

The results showed that regosol soil media with manure and charcoal affected growth in height, diameter, wet weight, total dry weight, and shoot-root ratio of the seedlings. This is because the application of a mixture of manure 
and charcoal results in the addition of N, P, K. This agrees with Pasaribu (2017), in which these elements function to nourish green leaves, increase leaf green, increase protein levels in the form of plant bodies, accelerate growth. Seedling roots accelerate and strengthen plant growth, accelerate the synthesis (formation) of carbohydrate substances in plants, and strengthen the whole plant body.

Furthermore, nitrogen is a macronutrient that is very important for growth for the metabolic process and the formation of amino acid compounds (Rentsch, et. al, 2007). If the nitrogen content of the soil increases, then crop production will tend to increase. Nitrogen bound in the form of nitrate is essential for the development of top plants. Increasing the growth media with the addition of manure and charcoal can increase the nitrogen content. Therefore, the high growth of $P$. merkusii on this medium was better than on soil media.

The application of a mixture of manure and charcoal in the seedling media in a particular concentration can increase the growth of mycorhizae and the growth of seedlings. This is following the opinion of (Ogawa (1992), who stated that charcoal might be suitable for the development of nitrogenfixing bacteria, Rhizobium, and VAM fungi, as well as for the growth of plant roots charcoal is generally porous, alkaline, absorbent. High and good aeration and can stimulate plant growth. Similarly, Norris and Date (1976) in Stowes and Elkan (1981), stated that the application of charcoal to the regosol soil medium in $P$. merkusii seedlings meant an opportunity to increase the utilization of the Rhizobium legume symbiosis because of the increase in $\mathrm{pH}$ and soil porosity. Legume simbiose - Rhizobium can increase soil nitrogen content through biological anchoring in root nodules. Legume symbiosis - Rhizobium can produce 100-200 N/ha/yr.

According to Hanover (1976), optimal growth acceleration is determined by photoperiod and other factors such as water, nutrients, $\mathrm{CO}_{2}$, and the type of growing media. If these factors work following the needs of the plant, then optimal growth will be achieved.

The most significant increase in average height occurred in media D (50\% regosol soil $+25 \%$ manure $+25 \%$ charcoal powder), which was $4.35 \mathrm{~cm}$, followed by media $\mathrm{F}$ by $3.85 \mathrm{~cm}$, and media B $3.85 \mathrm{~cm} .3 .1 \mathrm{~cm}$, which means a significant increase compared to media A (control) of $148.57 \%, 12 \%$, and $77.14 \%$. These results showed that applying a mixture of manure and charcoal powder with a particular concentration on the regosol soil seedling medium increased the seedling height and made a significant difference with the control medium. This is in line with the 
opinion (Sarira et al., 2020) that manure treatment significantly affects plant growth and agricultural yields. Besides that, it can also improve soil physical properties, namely soil structure, soil aggregate ability, and has no significant effect on all plant ages at height. plants, number of filled pods, wet weight of harvest, seed production (Purba, 2019), and manure and charcoal will improve the environment (Wasis, 2020).

Improvements in growing media will cause increased nutrient content. Therefore, the height of $P$. merkusii seedlings was better than the soil medium (control). In addition, with the improvement of the media, the $\mathrm{pH}$ of the media in the regosol soil will be higher (Sastriana, 2016); as a consequence, the availability of nutrients will be higher.

In $\mathrm{E}$ media $(25 \%$ regosol soil $+25 \%$ manure $+50 \%$ charcoal powder) and $\mathrm{C}$ media (50\% regosol soil $+50 \%$ charcoal powder), root growth was seen, so that the application of water and nutrients was not perfect. The findings of Devonald (1982) cited by Ogawa (1992) on peanut plants, showed that charcoal inhibited the growth of seedling root growth both in number of roots and in small size (Hutami, 2006), but organic growing media in the form of husk charcoal, sheep dung, cocopeat, and soil have a very significant effect on plant height (Asroh et al., 2020) if the application charcoal of excessive into the media that can poison the media (Widiastoety
$\&$ Marwoto, 2004) and have a negative impact on plants (Fajri et al., 2020).

$P$. merkusii seedlings has the most significant increase in average diameter was in media D, which was $0.55 \mathrm{~mm}$, followed by media F (0.55 mm), B (0.49 mm), and E (0.49 $\mathrm{mm})$, meaning an increase of $83,3 \%$ and $63.3 \%$ compared to medium A (control).

Only seedlings with larger diameters have a greater chance of survival in the field than smaller diameter seedlings (Alrasjid, 1980), (Setiadi, 2018). In Media E (25\% regosol soil $+25 \%$ manure $+50 \%$ charcoal powder) and $\mathrm{C}$ media $(50 \%$ regosol soil $+50 \%$ charcoal powder), growth tends to be depressed and imperfect, and this is due to imperfect root growth so that it affects nutrient absorption. and water needed for growth.

From the statistical test results on the fresh weight of seedlings, it shows that the use of a mixture of $50 \%$ regosol soil $+25 \%$ manure $+25 \%$ charcoal powder (media D) actually increased the fresh weight of seedlings, while the use of a mixture of $50 \%$ regosol soil + charcoal powder 50\% (media C) and a mixture of $2 \%$ regosol soil $+25 \%$ manure $+50 \%$ charcoal powder (media E), resulting in low seedling fresh weight.

The increase in the fresh weight of the seedlings was due to the increased growth of the seedlings, both in height and diameter. This is possible due to the influence of charcoal powder and manure containing 
nutrients, increasing the number of roots, able to increase the absorption of nutrients and water. The small fresh weight of the seedlings mentioned above was because of the suppressed growth of the seedlings due to the excessive amount of charcoal powder affecting root growth.

The total dry weight of the seedling showed the ability of the seedling to take the nutrients available in the seedling medium. In P. merkusii seedlings, the largest total dry weight was produced by medium $\mathrm{D}$, which was 0.035 grams (an increase of 180\% compared to the control). The size of the growth directly determines the total dry weight of seedlings. If the seedling grows well, it will obtain a large dry weight, meaning that the seedling can absorb the nutrients contained in the media, then through a metabolic process, it is converted and arranged into new cells (e.g., leaves, stems, twigs, flowers, fruit, etc.). Roots and so on experience an increase, addition, and enlargement of cells/fast growth.

The amount of shoot-root ratio is determined by shoot and root development. There is an interdependence between shoots and roots, where roots function to absorb nutrients, and shoots (stems, branches, and leaves) process mineral nutrients into valuable materials for growth and distribute them to other organs. In general, root growth will be followed by shoot growth (Firmansyah, 2019).
This study (attachment 14) showed that the highest average shoot-root ratio was found in media A of $2.94 \mathrm{~g}$. This means that the top of the seedling in dry weight has a very large value compared to the bottom dry weight.

In general, seedlings with too high a shoot-root ratio will have a lower ability to survive in the field than seedlings with a low root-shoot ratio (Chirino et.al., 2008). If the shoot-to-root ratio is high, the shoot portion is more significant than the root portion. This will cause transpiration in the shoot to be more outstanding while the seed supply will die. Good seeds usually have a shoot-root ratio between 2-5 (Alrasjid, 1972). The root shoot ratio describes the balance between the growth of the above-ground part of the seedling and the root part (Luturmas \& Mansur, 2017). However, each species has a different shootroot ratio and must meet the minimum height requirements to adapt to the field quickly.

\section{CONCLUSION}

Based on the results of calculations and analysis in this study, it can be concluded that the composition of $50 \%$ regosol soil mixed media with a mixture of $25 \%$ manure and $25 \%$ charcoal powder (media D) can significantly increase the average growth of seedling height by $4.35 \mathrm{~cm} \quad(148,57 \%$ compared control). Furthermore, the average seedling diameter was $0.55 \mathrm{~mm}(83,3 \%$ compared control). 
However, the difference in the mixed media composition did not have a significant effect on fresh weight, total dry weight, and shoot root ratio of Pinus merkusii seedlings. However, media D (50\% regosol soil $+25 \%$ manure + charcoal powder $25 \%$ ) increased the average fresh weight of seedlings by $0.22 \mathrm{~g}$ (185.71\% compared control), average total dry weight by $0.035 \mathrm{~g}$ (180\% compared control), and shoot-root ratio by 1,26 , shows a more balanced seedling between the shoots and roots compared to medium A (control).

\section{ACKNOWLEGDEMENT}

The author would like to thank the student participants of the study for their time and effort.

\section{REFERENCES}

Adisubroto, S. \& Priakusuma, S. (1985). Nursery Establishment Practices of Acacia mangium wild. Agency for Forestry Research and Development. Journal of Forestry Research an Development Bogor

Alrasjid, H. (1972). Teknik Persemaian dan penanaman di jepang. Laporan No. 142.

Asroh, A., Patimah, T., Meisani, ND, Irawan, R., \& Atabany, A. (2020). Penambahan arang sekam, kotoran domba dan cocopeat untuk media tanam. Jurnal Pusat Inovasi Masyarakat (PIM), 2(Khusus 1), 75-79.

Chirino, E., Vilagrosa, A., Hernández, E. I., Matos, A., \& Vallejo, V. R. (2008). Effects of a deep container on morpho-functional characteristics and root colonization in Quercus suber L. seedlings for reforestation in Mediterranean climate. Forest Ecology and Management, 256(4), 779-785.

Fachreza, A. (2021). Survival Pohon-Pohon Di Dalam Tegakan Pinus merkusii Pada Hutan Pendidikan Universitas Hasanuddin Setelah
4 Tahun Mengalami Kebakaran. Doctoral dissertation: Universitas Hasanuddin.

Fajri, K., Nopsagiarti, T., \& Okalia, D. (2020). Respon pertumbuhan eksplan jeruk nipis (Citrus aurantifolia L) dengan pemberian berbagai konsentrasi ekstrak pisang raja dan arang aktif pada media Ms. Green Swarnadwipa: Jurnal Pengembangan Ilmu Pertanian, 9(2), 230-241.

Firmansyah, MDWI (2019). Pertumbuhan Dan Hasil Tanaman Sorgum (Sorghum bicolor (L.) Moench) Varietas Numbu, Kawali, Super 2 Dan Suri 4 Agritan Di Kebun Percobaan $U M G$ : Universitas Muhammadiyah Gresik.

Hutami, S. (2006). Penggunaan arang aktif dalam kultur in vitro. Berita Biologi, 8(1), 83-89.

Hayati, M., \& Hayati, M. (2015). Pengaruh Media Simpan dan Lama Penyimpanan terhadap Viabilitas Benih dan Pertumbuhan Semai Mahoni (Swietenia mahagoni (L.) Jacq). Doctoral dissertation: Universitas Mataram.

Kuswiyono, D., \& Qomar, N. (2020). Analisis perubahan tutupan lahan di areal Iuphhk-Re Pt. Gemilang Cipta Nusantara Kabupaten Pelalawan. EcoNews, 3(2), 45-48.

Luturmas, FYR, \& Mansur, I. (2017). Efektifitas fungi mikoriza arbuskula (fma) serta pupuk nitrogen dan fosfat terhadap pertumbuhan semai jabon (Anthocephallus cadamba Roxb.). Jurnal Silvikultur Tropika, 8(1), 20 25 .

Pasaribu, S. (2017). Pengaruh Dosis Pupuk Kompos Gulma Siam Terhadap Pertumbuhan dan Hasil Tiga Varietas Bawang Merah. Universitas Mercu Buana Yogyakarta.

Purba, C. (2019). Respon Pemberian Pupuk NPK dan Arang Tempurung Kelapa terhadap Pertumbuhan dan Produksi Tanaman Kacang Tanah (Arachis hypogaea L.)". Universitas HKBP Nommensen.

Rentsch, D., Schmidt, S., \& Tegeder, M. (2007). Transporters for uptake and allocation of organic nitrogen compounds in plants. FEBS letters, 581(12), 2281-2289.

Sallata, M. K. (2013). Pinus (Pinus merkusii Jungh et de Vriese) dan keberadaannya di Kabupaten Tana Toraja, Sulawesi Selatan. Buletin Eboni, 10(2), 85-98.

Sarira, A., Tambing, Y., \& Lasmini, SA (2020). 
Aplikasi komposisi media tanam dan pupuk kandang pada pertumbuhan dan hasil tanaman temulawak (Curcuma xanthorrhiza Roxb.). AGROTEKBIS: E-JURNAL ILMU PERTANIAN, 8(3), 658-667.

Sastriana, E. (2016). Pengaruh Dosis Pupuk N (Nitrogen) terhadap Kandungan Protein Kasar dan Serat Kasar Rumput Gajah Cv. Mott pada Tanah Regosol. Doctoral dissertation: Universitas Mataram.

Setiadi, D. (2018). Keragaman Genetik Araucaria Cunninghamii Sumber Asal Benih Kepulauan Papua pada Pertumbuhan Di Tingkat Semai.
Proceeding Biology Education Conference: Biology, Science, Enviromental, and Learning, 15(1), 785-790.

Wasis, B. (2020). Teknik Pemberian Arang Charcoal Application Techniques. Makalah pengayaan materi mata kuliah Pengelolaan Nutrisi Hutan. Departemen Silvikultur: Fakultas Kehutanan dan Lingkungan IPB University, Bogor

Widiastoety, D., \& Marwoto, B. (2004). Pengaruh berbagai sumber arang dalam media kultur in vitro terhadap pertumbuhan plantlet oncidium. Jurnal Hortikultura, 14(1), 1-5. 